\title{
An Efficient Strategy for Reducing Head-of-Line Blocking in Fat-Trees
}

\author{
Jesus Escudero-Sahuquillo ${ }^{1}$, Pedro Javier Garcia ${ }^{1}$, \\ Francisco J. Quiles $^{1}$, and Jose Duato ${ }^{2}$ \\ 1 Dept. of Computing Systems, University of Castilla-La Mancha, Spain \\ \{jescudero,pgarcia, paco\}adsi.uclm.es \\ 2 Dept. of Computer Engineering, Technical University of Valencia, Spain \\ jduato@gap.upv.es
}

\begin{abstract}
The fat-tree is one of the most common topologies for the interconnection networks of PC Clusters which are currently used for high-performance parallel computing. Among other advantages, fat-trees allow the use of simple but very efficient routing schemes. One of them is a deterministic routing algorithm that has been recently proposed, offering similar (or better) performance than Adaptive Routing while reducing complexity and guaranteeing in-order packet delivery. However, as other deterministic routing proposals, this deterministic routing algorithm cannot react when high traffic loads or hot-spot traffic scenarios produce severe contention for the use of network resources, leading to the appearance of Head-Of-Line (HOL) blocking, which spoils network performance. In that sense, we present in this paper a simple, efficient strategy for dealing with the HOL blocking that may appear in fat-trees with the aforementioned deterministic routing algorithm. From the results presented in the paper, we can conclude that, in the mentioned environment, our proposal considerably reduces HOL blocking without significantly increasing switch complexity and required silicon area.
\end{abstract}

Keywords: Interconnection Networks, Fat-trees, Deterministic Routing, Head-of-line Blocking.

\section{Motivation}

High-performance interconnection networks are currently key elements for many types of computing and communication systems: massive parallel processors (MPPs), local and system area networks, IP routers, networks on chip, and clusters of PCs and workstations. In such environments, the performance achieved by the whole system greatly depends on the performance the interconnection network offers, especially when the number of processing and/or storage nodes is high. On its side, network performance depends on several issues (topology, routing, switching, etc.) which should be carefully considered by interconnect designers in order to obtain the desired low packets latencies and high network bandwidth.

However, network design decisions are not actually taken based only on the achieved performance, but also on other factors, like network cost and power consumption.

P. D'Ambra, M. Guarracino, and D. Talia (Eds.): Euro-Par 2010, Part II, LNCS 6272, pp. 413-427, 2010.

(c) Springer-Verlag Berlin Heidelberg 2010 
In fact, a clear trend on interconnect researching is to propose cost-effective techniques, which allow to obtain good performance while minimizing network resource requirements. Obviously, cost-effective solutions are especially relevant for the commercial high-speed interconnects (Myrinet[1], Infiniband[2], etc.) used for building high-performance clusters, since they must satisfy, at affordable cost, the growing performance demands of cluster designers and users (many of the most powerful parallel computers are currently cluster-based machines [3]).

In that sense, the fat-tree [4] has become one of the most popular network topologies since it offers (among other properties) a high communication bandwidth while minimizing the required hardware. Consequently, many interconnection networks in current clusters and MPPs (for instance, the Earth Simulator[5]) are fat-trees.

Additionally, the fat-tree pattern eases the implementation of different efficient routing algorithms, either deterministic (packets follow a fixed path between source and destination) or adaptive (packets may follow several alternative paths). In general, adaptive routing algorithms are more difficult to implement than deterministic ones and present problems regarding in-order packet delivery and deadlock-freedom, but it has been traditionally assumed that they better balance traffic, thus achieving higher throughput. However, a recently proposed deterministic routing algorithm for fat-trees [6] achieves a similar or better throughput than adaptive routing, thanks to a smart balance of link utilization based on the properties of the fat-tree pattern. This algorithm can be implemented in a cost-effective way by using Flexible Interval Routing [7] (FIR), a memoryefficient generic routing strategy. Summing up, this algorithm offers the advantages of deterministic routing (simple implementation, in-order packet delivery, deadlockfreedom) while reaching the performance of (or even outperforming) adaptive routing. Hereafter, this algorithm will be referred to as DET.

However, the DET routing algorithm, like other deterministic proposals, is unable to keep by itself its good performance level when packet contention appears. Contention happens in a switch when several packets, from different input ports, concurrently require access to the same output port. In these cases, only one packet can cross while the others will have to wait until the required output port becomes free, thus their latency increases. Moreover, when contention is persistent (in this case, it is usually referred to as congestion), a packet waiting to cross will prevent other packets stored behind in the same input buffer from advancing, even if these packets request a free output port, thus their latency increases and switch throughput drops. This phenomenon is known as Head-of-Line (HOL) blocking, and may limit the throughput of the switch up to about $58 \%$ of its peak value [8]. Of course, high traffic loads and hot-spot situations favor the appearance of contention and HOL blocking, and consequently in these scenarios network performance suffers degradation. In order to solve that problem, many mechanisms have been proposed.

In that sense, as modern high-speed interconnects are lossless (i.e. discarding blocked packets is not allowed), the most common approach to deal with HOL blocking is to have different queues at each switch port, in order to separately store packets belonging to different flows. This is the basic approach followed by several techniques that, on the other hand, differ in many aspects, basically in the required number of queues and in the policy for mapping packets to queues. For instance, Virtual Output Queues at network 
level (VOQnet)[9] requires as many queues per port as end-nodes, mapping each packet to the queue assigned to its destination. This solution guarantees that packets addressed to different destinations never share queues, thus completely eliminating HOL blocking, but, on the other hand, it is too costly in terms of the silicon area required to implement all these queues per port in medium or large networks. On the contrary, other techniques like Virtual Output Queues at switch level (VOQsw)[10], Dynamically Allocated Multi-Queues (DAMQs)[11] or Destination-Based Buffer Management (DBBM)[12] use far fewer queues per port than VOQnet. Although these techniques map packets to queues according to different "static" (i.e. independent of traffic conditions) criteria, all of them allow packets belonging to different flows to share queues, thus they just partially reduce HOL blocking. In contrast with the "static" queue assignment of the previously mentioned techniques, both Regional Explicit Congestion Notification (RECN)[13], [14] and Flow-Based Implicit Congestion Management (FBICM) [15] detect which flows cause HOL blocking and dynamically assign queues to separate them from others. Although this approach quite efficiently eliminates HOL blocking without using many queues, it requires specific mechanisms to detect congested flows and additional resources (basically, Content-Addressable Memories, CAMs) to keep track of them. In conclusion, it seems that an effective HOL blocking elimination would imply to considerably increase switch cost and/or to introduce significant complexity.

On the contrary, we think that, if the queue assignment criterion exploits the properties of both network topology and routing scheme, it would be possible to quite effectively eliminate HOL blocking without using too many queues per port and without introducing significant complexity. In that sense, we propose in this paper an efficient HOL blocking elimination technique for fat-trees which use the DET routing algorithm. Our proposal uses a reduced set of queues per port, mapping packets to these queues according to the traffic balance the routing algorithm performs. As it is shown in the paper, by linking queue assignment to the routing algorithm, our proposal reduces HOL blocking probability with the minimum number of resources, thus reducing resource requirements of other generic HOL blocking elimination techniques while achieving similar (or even better) performance. Moreover, as queue assignment is static in our proposal, it does not introduce additional complexity, as dynamic approaches do. Summing up, we propose a cost-effective and simple technique for eliminating HOL blocking in a cost-effective interconnect configuration (fat-trees using the DET routing algorithm). As the proposed queue assignment is based on traffic balance, and this balance is based on a smart distribution of destinations among output ports, we call our proposal OutputBased Queue Assignment (OBQA).

The rest of the paper is organized as follows. In Section 2 we summarize the deterministic routing algorithm for fat-trees which is the base of our proposal. Next, in Section 3 , the basics of our OBQA proposal are presented. In Section 4 , OBQA is compared in terms of performance and resource needs to previously proposed techniques which also reduce HOL blocking. Finally, in Section 5, some conclusions are drawn.

\section{Efficient Deterministic Routing in Fat-Trees}

Theoretically, a fat-tree topology [4] is an indirect bidirectional interconnection network consisting of several stages of switches connected by links, forming a complete tree 


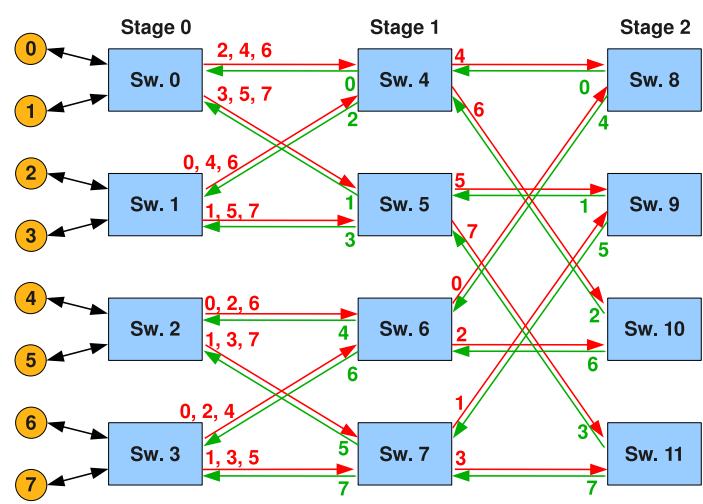

Fig. 1. Eight node 2-ary 3-tree with DET routing algorithm

which gets "thicker" near the "root" switch (transmission bandwidth between switches is increased by adding more links in parallel as switches become closer to the root), and whose "leaves" (switches at the first stage) are the places where processors are located. However, as this theoretical scheme requires that the number of ports of the switches increases as we get closer to the root, its implementation is actually unfeasible. For this reason, some equivalent, alternative implementations have been proposed that use switches with constant radix [16]. In [17] a fat-tree definition is given for embedding all the topologies quoted as fat-trees. Among these, we focus on $k$-ary $n$-trees, a parametric family of regular multistage interconnection networks (MINs) [18], where bandwidth between switches is increased as we go towards the root by replicating switches.

A $k$-ary $n$-tree topology is defined by two parameters: $k$, which represents the arity or number of ports connecting a switch to the next or previous stage, and $n$, which represents the number of stages of the network. A $k$-ary $n$-tree is able to interconnect $k^{n}$ nodes and includes $n k^{n-1}$ switches. Figure 1 shows a 2 -ary 3 -tree connecting 8 processing nodes with 12 switches.

In such topologies, a minimal source-destination path can be easily found by going from source to one of the nearest common ancestors of both source and destination ("upwards" direction) and then turning towards destination ("downwards" direction). Note that when advancing upwards, several paths are possible (thus making possible adaptive routing), but when advancing downwards, only a single path is available. Taking that into account, adaptivity is limited to the upwards path, where any switch can select any of its upwards output ports for forwarding packets towards the next stage, but this is enough for allowing adaptive routing algorithms based on different criteria. For instance, it's possible to use a round-robin policy for selecting output ports, in order to balance traffic inside the network and thus improving performance. However, adaptive routing algorithms introduce out-of-order packet delivery, as packets may cross different paths between the same source-destination pair.

By contrast, a deterministic routing algorithm able to balance traffic as well as adaptive routing schemes would solve the out-of-order delivery problem without losing performance. Such deterministic routing algorithm (as mentioned above referred to as 


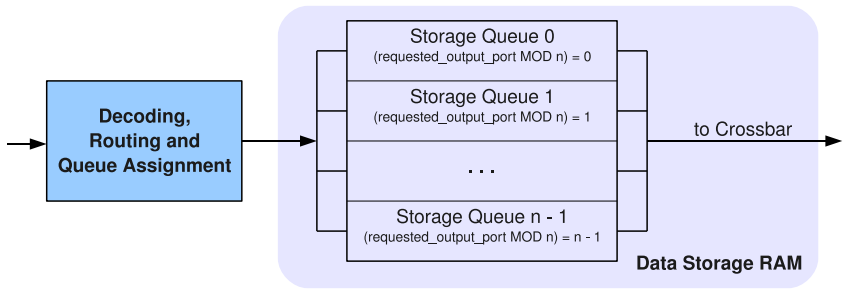

Fig. 2. OBQA logical input port organization

DET) is proposed in [6], and it reduces the multiple upwards paths into a single one for each source-destination pair without unbalancing network link utilization (so, all the links of a given stage are used by a similar number of paths). This is accomplished by shuffling consecutive destinations at each switch in the upwards direction. That means that consecutive destinations are distributed among the upwards output links, reaching different switches in the next stage.

Figure 1 also shows the destination distribution proposed by the deterministic routing algorithm in a 2-ary 3-tree network. Note that each link is labeled 1 with its assigned destinations (that is, with the destinations of packets that will be forwarded through it). It can be seen that, by shuffling destinations as proposed, packets crossing a switch are addressed to a number of destinations that decrease as the stage of the switch increases (that is, the higher the stage, the lower number of destinations the switch deals with). In fact, each switch of the last stage receives packets addressed only to two destinations, and packets destined to each one are forwarded through a different downwards link (e.g. switch 8 receives packets only for destinations 0 and 4 , and they are forwarded downwards through different output ports). Note also that packets addressed to the same destination reach the same switch at the last stage, then following a unique downwards path. That means that, when going downwards, a packet share links only with packets addressed to the same destination. In this way, the mechanism distributes the traffic destined to different nodes and thus traffic is completely balanced (that is, both upwards and downwards links at a given stage are used by the same number of paths).

In [6], more details about this deterministic routing algorithm for fat-trees can be found, including a possible implementation using Flexible Interval Routing [7] and an evaluation of its performance. This evaluation shows that this deterministic routing algorithm equals adaptive routing for synthetic traffic patterns (while being simpler to implement), but it outperforms adaptive routing by a factor of 3 when real applications are considered. Summing up, this deterministic algorithm is one of the best options regarding routing in fat-trees, since it reaches high performance while being cost-effective.

However, as this deterministic routing proposal considers only switches with singlequeue ports, its performance drops when contention situations cause Head-Of-Line blocking in the single queues, thus some mechanism should be used to help this routing algorithm to guarantee a certain performance level even in those situations.

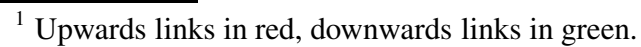

${ }^{2}$ All in all, it's not in the scope of the DET routing algorithm to solve the HOL blocking problem.
} 


\section{OBQA Description}

In this section we describe in depth our new proposal for reducing HOL blocking in fat-trees. As mentioned above, we call our proposal Output-Based Queue Assignment (OBQA), since it assigns packets to queues depending on its requested output port, thus taking advantage of the traffic balance the DET routing algorithm performs. In the following paragraphs, we firstly detail the assumed memory organization at each switch port. Next, we explain the OBQA basics and how it "fits" with the DET routing algorithm in order to efficiently eliminate HOL blocking.

Figure 2 depicts a diagram of the assumed queue scheme at input port 3 . As can be seen, a RAM is used to store incoming packets, this memory being statically divided into a reduced set of queues of fixed size. The exact number $(n)$ of queues may be tuned, but, as one of our objectives is to reduce the queue requirements of other techniques, we assume that $n$ is always smaller than switch radix (as we explain in Section 4 a value of $n$ equal to or lower than half of switch radix is enough for quite efficiently eliminating HOL blocking). In order to calculate the queue in which each incoming packet must be stored, OBQA performs a simple modulo-mapping operation between the output port requested by the packet and the number of queues per port, thus:

assigned_queue_number = requested_output_port MOD number_of_queues

This queue assignment is the key of OBQA functionality. As a first benefit, packets requesting the same output port (which in fact may be addressed to different destinations) are stored in the same input queue, thus if that output port becomes congested, packets in other queues do not suffer HOL blocking. Moreover, taking into account that we assume the use of the DET routing algorithm, the number of destinations a switch deals with is lower as higher the stage (as can be seen in Figure 1), thus the number of destinations assigned to each OBQA queue is lower as higher the stage. Therefore, the higher the stage, the less destinations share queues, thus decreasing HOL-blocking probability. In fact, both HOL blocking whose origin is contention inside switch and HOL blocking created by contention in other switches are reduced.

Figure 3 depicts an example of OBQA operation in a portion of a 2-ary 3-tree (see Figure 1) consisting of several switches in different stages. Note that switch radix is 4 , switch ports being numbered from P0 to P3. We assume the number of queues per port is 2 , although only queues at port 0 in every switch are shown. Inside the queues, packets are labeled with their own destination. As in Figure 1, routing information are depicted besides the output ports, indicating which destinations are assigned to each output port (upwards destinations are colored in red, while downwards ones in green).

In the example, packets addressed to all destinations are injected by the end-node connected to port 0 of switch 0 . When received at switch 0 , these packets are stored in their corresponding input queue, which is calculated by using the aforementioned modulo-mapping function. For instance, packets addressed to destinations 1, 3, 5 or 7 are stored in queue 1 because, for these destinations, the requested output ports are either $\mathrm{P} 1$ or $\mathrm{P} 3$, then in all these cases requested_output_port MOD $2=1$. Analogously, packets addressed to destinations 2,4 or 6 are stored in queue 0 .

\footnotetext{
${ }^{3}$ Hereafter, for the sake of simplicity, we assume an Input-Queued (IQ) switch architecture, but note OBQA could be also valid for Combined Input and Output Queued (CIOQ) switches.
} 


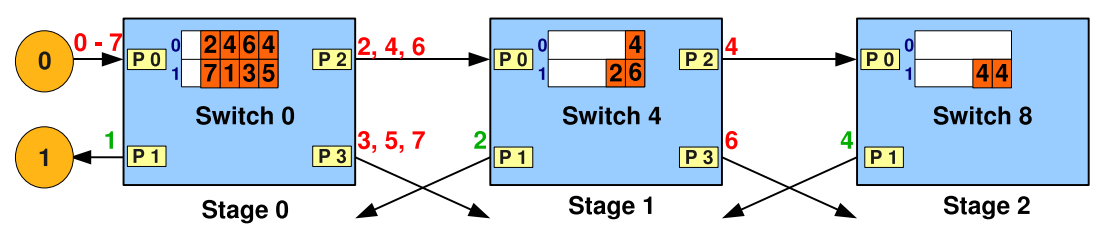

Fig. 3. OBQA operation example $(4 \times 4$ Switches $)$

At the next stage, the number of destinations present in switch 4 are lower, because the DET routing algorithm smartly balances traffic between network links. For instance, port 0 of switch 4 only receives packets addressed to destinations 2,4 and 6 , and the routing algorithm again balances traffic among all possible output ports. At this point, packets addressed to destination 4 request $\mathrm{P} 2$, thus being stored in queue 0 , while packets addressed to destinations 2 or 6 respectively request $\mathrm{P} 1$ and $\mathrm{P} 3$, thus being stored in queue 1 . Note that at this stage, the number of destinations sharing each queue is lower than in the former stage, then HOL-blocking probability is reduced.

At switch 8 , port 0 only receives packets addressed to destination 4 . This switch is at the highest stage and, for this reason, the number of destinations the switch deals with is minimal, thus HOL-blocking is completely eliminated at this stage. Finally, as the DET routing algorithm implies that downwards paths are exclusively used by packets addressed to the same destination, HOL-blocking situations in downwards paths are not possible.

Summing up, OBQA smartly exploits both $k$-ary $n$-trees benefits and the DET routing algorithm properties for progressively reducing HOL-blocking probability along any path inside the fat-tree. As this is achieved with a reduced set of queues per port, OBQA can be considered a cost-efficient technique for HOL-blocking elimination in fat-trees.

In the next section, we evaluate OBQA in comparison with other schemes proposed for reducing HOL blocking, in terms of performance and memory requirements.

\section{Performance Evaluation}

In this section we present the evaluation of the OBQA mechanism, based on simulation results showing network performance when OBQA or other HOL-blocking elimination technique are used. The simulation tool used in our experiments is an ad-hoc, eventdriven simulator modeling interconnection networks at cycle level. In the next sections we firstly describe the simulated scenarios and modeling considerations used to configure all the experiments. Then, we show and analyze the simulation results.

\subsection{Simulation Model}

The simulator models different types of network topologies by defining the number of switches, end-nodes and links. In our experiments, we model fat-trees ( $k$-ary $n$-trees) with different network sizes and different switch radix values. In particular, we use the network configurations shown in Table 1 . 
Table 1. Evaluated network configurations

\begin{tabular}{|c|c|c|c|c|c|}
\hline$\#$ & Fat-Tree Size & Interconnection Pattern & $\#$ Switch radix & \#Switches (total) & \#Stages \\
\hline$\# 1$ & $64 \times 64$ & 4-ary 3-tree & 8 & 48 & 3 \\
\hline$\# 2$ & $256 \times 256$ & 4-ary 4-tree & 8 & 256 & 4 \\
\hline$\# 3$ & $256 \times 256$ & 16-ary 2-tree & 32 & 32 & 2 \\
\hline
\end{tabular}

For all network configurations, we use the same link model. In particular, we assume serial full-duplex pipelined links with 1GByte/s of bandwidth and 4 nanoseconds for link delay, both for switch-to-switch links as node-to-switch links.

The DET routing algorithm described in Section 2 has been used for all the network configurations in Table 1

The modeled switch architecture follows the IQ scheme, thereby memories are implemented only at switch input ports. However, memory size and organization at each input port depend on the mechanism used for reducing HOL-blocking. In that sense, for the purpose of comparison, the simulator models the following memory queue schemes:

- OBQA. A memory of $4 \mathrm{~KB}$ per input port is used, statically and equally divided among all queues in the input port. As described in Section 3, queues are assigned to a set of output ports (taking into account the routing algorithm, that means that a queue is assigned to a set of destinations) according to the aforementioned modulomapping function. We consider three values for the number of queues: 2, 4 or 8 .

- DBBM. As in the previous scheme, a memory of $4 \mathrm{~KB}$ per input port is assumed, statically and equally divided among all configured DBBM queues in the port. Each queue is assigned to a set of destinations according to the modulo-mapping function assigned_queue_number = destination MOD number_of_queues. We consider 4 or 8 queues per port for DBBM.

- Single Queue. This is the simplest case, with only one queue at each input port for storing all the incoming packets. Hence, there is no HOL-blocking reduction policy at all, thus this scheme allows to evaluate the performance achieved by the "alone" DET routing algorithm. $4 \mathrm{~KB}$ memories are used for this case.

- VOQ at switch level (VOQsw). $4 \mathrm{~KB}$ memories per input port are used, statically and equally divided into as many queues as switch output ports, in order to store each incoming packet in the queue corresponding to its requested output port. As the number of queues equals switch radix, 8 queues are used for network configurations \#1 and \#2, and 32 queues for network configuration \#3.

- VOQ at network level (VOQnet). This scheme, although the most effective one, requires a greater memory size per port, because the memory must be split into as many queues as end-nodes in the network, and each queue requires a minimum size. Considering flow control restrictions, packet size, link bandwidth and link delay, we fix the minimum queue size to 512 bytes, which implies input port memories of 32 $\mathrm{KB}$ for the $64 \times 64$ fat-tree and $128 \mathrm{~KB}$ for the other (larger) networks. Note that this scheme is actually almost unfeasible, thus it is considered only for showing theoretical maximum efficacy in HOL-blocking elimination 4 .

\footnotetext{
${ }^{4}$ As both RECN and FBICM have been reported to obtain a performance level similar to VOQnet, we consider redundant to include these techniques in the comparison.
} 
Table 2. Synthetic traffic patterns used in the evaluation

\begin{tabular}{|c|c|c|c|c|c|c|c|c|}
\hline & \multicolumn{3}{|c|}{ Random Traffic } & \multicolumn{4}{c|}{ Hot-Spot Traffic } \\
\hline Traffic case & \# Sources & Destination & Generation rate & \# Sources & Destination & Generation rate & Start time & End time \\
\hline \# 1 & $100 \%$ & random & incremental & $0 \%$ & - & - & - & - \\
2 & $75 \%$ & random & $100 \%$ & $25 \%$ & 123 & $100 \%$ & $250 \mu \mathrm{s}$ & $300 \mu \mathrm{s}$ \\
\hline
\end{tabular}

Notice that although memory requirements are different for each policy, we use the same memory amount per port (4 KB), except in VOQnet scheme. Later, we analyze the minimum memory requirements for each mechanism, thus putting results in this section in the right context.

Regarding message switching policy, we assume Virtual Cut-Through. Moreover, in all the switches, the flow control policy is a credit-based mechanism at the queue level. Packets are forwarded from input queues to output queues through a multiplexed crossbar, modeled with a speedup of 1 (link bandwidth is equal to crossbar bandwidth).

The end-nodes are connected to switches through Input Adapters (IAs). Every IA is modeled with a fixed number of admittance queues (as many as destinations in the network, in order to avoid HOL-blocking before packet injection), and a variable number of injection queues, which follow the same selected scheme as queues at input port memories. A generated message is completely stored in a particular admittance queue assigned to its destination. Then, the stored message is packetized before being transferred to an injection queue. We use 64-byte packets.

Regarding traffic loads, we use both synthetic traffic patterns (in order to simulate ideal traffic scenarios) and storage area network (SAN) traces. The synthetic traffic patterns are described in Table 2. For uniform traffic (traffic case \#1) each source injects packets addressed to random destinations. We range injection rate from $0 \%$ up to $100 \%$ of the link bandwidth. In addition, a simple, intensive hot-spot scenario (traffic case \#2) is defined, in order to create heavy congestion situations within the network. In this case congestion has been generated by a percentage of sources $(25 \%)$ injecting packets always addressed to the same destination, while the rest of sources $(75 \%)$ inject packets to random destinations. Note that random packet generation rate is incremental in case $\# 1$, thus increasing the traffic rate from $0 \%$ up to $100 \%$ of link bandwidth. By contrast, traffic pattern \#2 has been used to obtain performance results as a function of time, in order to show the impact of a sudden congestion situation. These synthetic traffic patterns have been applied to network configurations \#2 and \#3.

On the other hand, we use real traffic traces provided by Hewlett-Packard Labs. They include all the I/O activity generated from 1/14/1999 to 2/28/1999 at the disk interface of the cello system. As these traces are eleven years old, we apply several time compression factors to the traces. Of course, only results as a function of time are shown in this case. Traces are used for network configuration \#1.

Finally, although the simulator offers many metrics, we base our evaluation on the ones usually considered for measuring network performance: network throughput (network efficiency when normalized) and packet latency. Therefore, in the following subsections we analyze, by means of these metrics, the obtained network performance. 


\subsection{Results for Uniform Traffic}

Figure 4 depicts the simulation latency results as a function of traffic load, obtained for fat-trees configurations \#2 (Figure 4(a)) and \#3 (Figure 4(b)) when traffic case \#1 (completely uniform traffic) is used.

As can be seen in Figure 4 (a), when the network is made of switches of radix 8 (configuration \#2), OBQA configured with 4 queues per port reaches the saturation point (the point where average message latency dramatically increases) for the same traffic load as VOQnet and VOQsw. Note that in this case VOQsw requires 8 queues per input port and VOQnet requires 256 queues. Thus, OBQA equals the maximum possible performance while significantly reducing the number of required queues. On its side, the DBBM scheme, which is also configured using 4 queues per input port, experiences a high packet latency, near the poorest result (the one of the Single Queue scheme). It can also be seen that, even when configured with just 2 queues, OBQA achieves better results than DBBM with 4 queues, although worse than VOQsw (however, note that the difference is around $12 \%$ while queues are reduced in $75 \%$ ). Note also that using OBQA increases dramatically (around 30\%) the performance achieved by the Single Queue scheme (that is, the performance achieved by the DET routing algorithm without HOL blocking elimination mechanism).

For a network with switches of radix 32 (Figure 4 (b)), OBQA configured with 8 queues per port reaches the saturation point for the same traffic load as VOQsw, which in this case requires 32 queues per port. Moreover, this traffic load is just $2 \%$ lower than the VOQnet saturation point, thus the number of queues per port could be reduced from 256 to 8 at the cost of a minimum performance decrease. Even if configured with 4 queues, OBQA reaches the saturation point at a load only $5 \%$ lower than VOQsw. Again, the Single Queue scheme and DBBM with 4 or 8 queues achieve very poor results. As already explained, that is what we should expect for the Single Queue scheme, since it does not implement any HOL blocking elimination mechanism. However, the reason for the poor behavior of DBBM is that its queue assignment policy does not "fit" the routing algorithm (on the contrary to OBQA), thus some DBBM queues are not efficiently used for eliminating HOL blocking. In fact, considering the routing algorithm, in some switches beyond the first stage, some DBBM queues are not used at all.

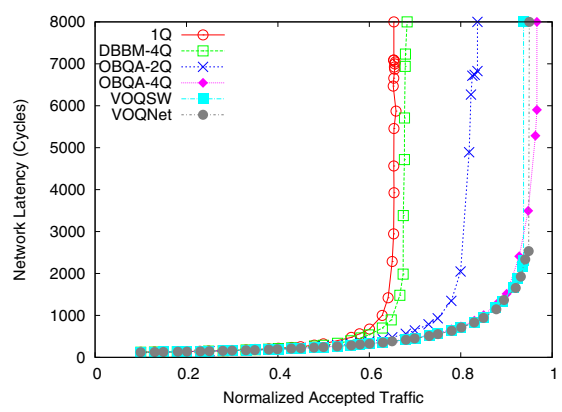

(a) Network Configuration \#2

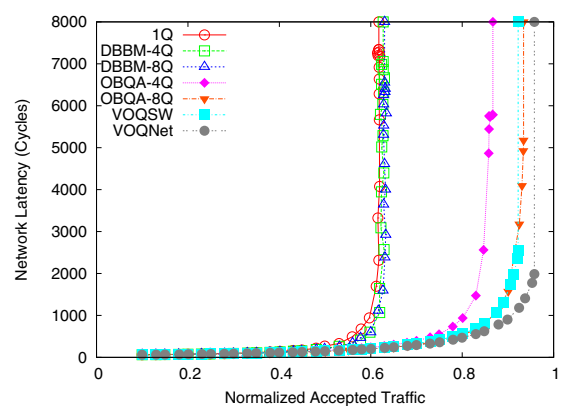

(b) Network Configuration \#3

Fig. 4. Network latency versus Accepted traffic. Uniform distribution of packet destinations. 
Therefore, we can conclude that, in a uniform traffic scenario, OBQA achieves a network performance similar to the VOQnet and VOQsw ones, while requiring far less queues per port (specifically, a maximum number of queues per port equal to half the switch radix). Moreover, OBQA clearly outperforms DBBM.

\subsection{Results for Hot-Spot Traffic}

In this subsection, we present in Figure 5 network efficiency results as a function of time, when synthetic traffic pattern \#2 is used in fat-tree configurations \#2 (Figure 5(a)) and \#3 (Figure 5(b)). As previously described, in this case $25 \%$ of end-nodes generate hot-spot traffic addressed to a single end-node (specifically, destination 123), whereas the rest of traffic is randomly distributed. Furthermore, the hot-spot generation sources are active only during 50 microseconds, after 250 microseconds from simulation start time, thus creating a sudden, eventual congestion situation.

As can be seen in Figure 5(a), for a network with 8-radix switches, the Single Queue scheme barely achieves a $5 \%$ of network efficiency when congestion appears. Likewise, DBBM (with 4 queues) performance decreases around $25 \%$ when congestion arises. Obviously, both queue schemes are dramatically affected by the HOL-blocking created by congestion, and don't recover their maximum theoretical performance during simulation time. OBQA with 4 queues per port and VOQsw (8 queues per port) achieve similar performances which are better than the DBBM one, decreasing around $20 \%$ when the hot-spot appears, thus they slightly improve DBBM behavior and completely outperform the Single Queue scheme. However, again OBQA requires half the queues than VOQsw while achieving similar performance. On its side, VOQnet achieves the maximum efficiency but requiring 256 queues. Similar results are achieved for a fattree made of 32-radix switches, as can be seen in Figure 5b), VOQsw (32 queues per port in this case) achieving the same results as OBQA with 8 queues per port. Note that in this case the performance level of OBQA with 8 queues is quite close to the maximum (VOQnet), and also that OBQA with 4 queues outperforms DBBM with 8 queues.

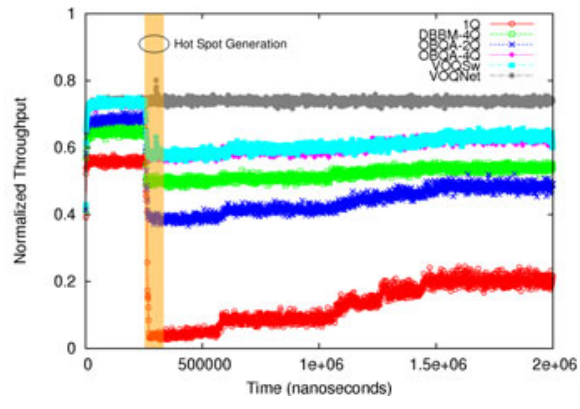

(a) Network Configuration \#2.

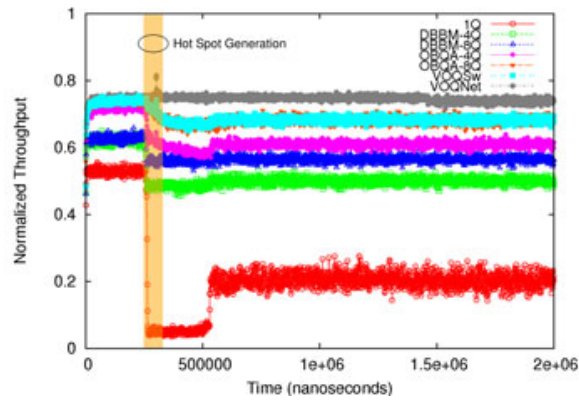

(b) Network Configuration \#3.

Fig. 5. Network efficiency (normalized throughput) versus Time. Hot-Spot traffic (25\% of packets are addressed to the same destination). 


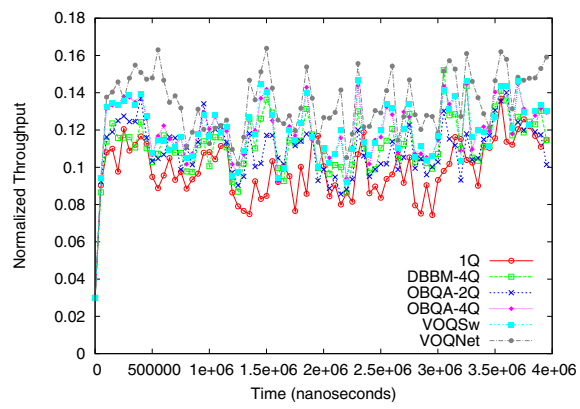

(a) Network Configuration \#1. FC=20.

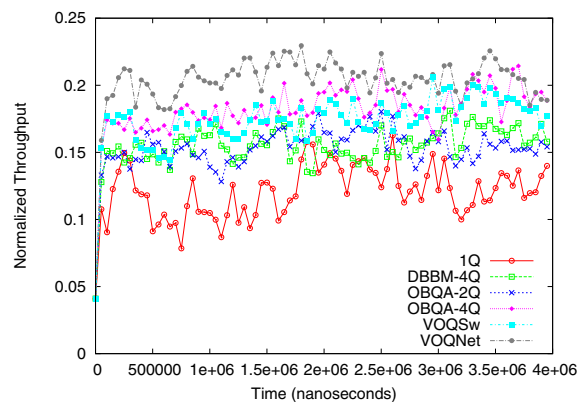

(b) Network Configuration \#1. FC=40.

Fig. 6. Network efficiency (normalized throughput) versus Time. Storage area network (SAN) traces with different compression factors.

Summing up, the analysis of hot-spot results leads to similar conclusions than the former (uniform traffic) analysis: OBQA reaches great performance with a number of queues per port equal to half or a quarter switch radix.

\subsection{Results for Traces}

Finally, we evaluate network performance when real traffic (the I/O traces described in section 4.1) are used as traffic load. In this case we use fat-tree configuration \#1 (64 nodes, switch radix is 8). Figure 6 a) shows results for a trace compression factor of 20. As can be seen, OBQA with 4 queues again achieves excellent efficiency, at the same level as VOQsw (8 queues), and they slightly outperforms DBBM (4 queues) and the Single Queue scheme. When a higher compression factor (40) is applied to the trace (Figure 6(b)), it can be seen that OBQA (4 queues), as VOQsw, more clearly outperforms DBBM, achieving in some cases a 30\% of improvement. Again, the Single Queue scheme achieves the poorest results (until $50 \%$ of the OBQA efficiency).

\subsection{Data Memory Requirements}

In this section, we compare the minimum data memory requirements of different switch organizations, as an estimation of switch complexity in each case. In particular, we consider the same HOL blocking elimination queue schemes modeled in the simulation tests.

Table 3 shows memory size and area requirements for each queue scheme at each switch input port. In order to help in the comparison, different number of queues per port have been evaluated for different schemes. For each case the minimum memory requirements have been computed assuming two packets per queue (64-byte packet size) and Virtual Cut-Through switching. All queue schemes can be interchangeably used by $8 \times 8$ and $32 \times 32$ switches, except the VOQsw ones. In particular, line \#3 corresponds to $32 \times 32$ switches, and line \#4 represents data for $8 \times 8$ switches. On the other hand, VOQnet requirements are shown for 256-node networks (line \#1) and for 
Table 3. Memory size and area requirements per input port for different queue schemes

\begin{tabular}{|l|c|c|r|c|}
\hline$\#$ & Queue scheme & \# Queues per port & Data Memory size & Data Memory Area per port $\left(\mathrm{mm}^{2}\right)$ \\
\hline 1 & $V O Q_{\text {net }}$ & 256 & 32768 bytes & 0.33291 \\
2 & $V O Q_{n e t}$ & 64 & 8192 bytes & 0.07877 \\
\hline 3 & $V O Q_{s w}$ & 32 & 4096 bytes & 0.03569 \\
4 & $V O Q_{s w}$ & 8 & 1024 bytes & 0.01412 \\
\hline 5 & $D B B M$ & 8 & 1024 bytes & 0.01412 \\
6 & $D B B M$ & 4 & 512 bytes & 0.00647 \\
\hline 7 & $O B Q A$ & 8 & 1024 bytes & 0.01412 \\
8 & $O B Q A$ & 4 & 512 bytes & 0.00647 \\
9 & $O B Q A$ & 2 & 256 bytes & 0.00359 \\
\hline
\end{tabular}

64-node networks (line \#2). Memory area has been estimated by means of the CACTI tool v5.3 [19] using its SRAM modeling. We assume SRAM memories with one read and one write port, $45 \mathrm{~nm}$ technology node, and 1 byte as readout value.

As can be seen, in general OBQA and DBBM present similar memory size and area requirements per port, although, as we have shown in previous sections, OBQA always outperforms DBBM when configured with the same number of queues (or even with less queues than DBBM in some scenarios). On its side, VOQsw requirements for $32 \times 32$ switches are much greater than the ones of any OBQA or DBBM schemes. For $8 \times 8$ switches, VOQsw requirements ( 8 queues) equal OBQA and DBBM requirements if configured with 8 queues. Note, however, that for this switch radix, OBQA with 4 queues always equals VOQsw performance, thus in this case OBQA requirements are actually lower than (half) the VOQsw ones. Finally, VOQnet needs a vast amount of memory storage as it demands many queues (as many queues as destinations in the network), and its required area is impractical in real implementations.

\section{Conclusions}

Currently, one the most popular high-performance interconnection network topologies is the fat-tree, whose nice properties have favored its use in many clusters and massive parallel processors. One of these properties is the high communication bandwidth offered with the minimum hardware, thus being a cost-effective topology. The special connection pattern of the fat-tree has been exploited by a deterministic routing algorithm which achieves the same performance as adaptive routing in fat-trees, whereas being simpler and thus cost-effective. However, the performance of that deterministic routing algorithm is spoiled by Head-Of-Line blocking when this phenomenon appears due to high traffic loads and/or hot-spot scenarios.

The HOL blocking elimination technique proposed, described and evaluated in this paper solves this problem, keeping the good performance of the aforementioned deterministic routing algorithm, even in adverse scenarios. Our proposal is called OutputBased Queue Assignment (OBQA), and it is based on using at each switch port a reduced set of queues, and on mapping incoming packets to queues in line with the routing algorithm. Specifically, OBQA uses a modulo-mapping function which selects 
the queue to store a packet depending on its requested output port, thus suiting the routing algorithm. From the results shown in this paper we can conclude that a number of OBQA queues per port equal to half (or even a quarter) switch radix is enough for efficiently dealing with HOL blocking even in scenarios with high traffic loads and/or hot-spots, especially in networks where switch radix is not high. In fact, OBQA outperforms previous techniques with similar queue requirements (like DBBM) and achieves similar (or slightly worse) performance than other techniques with much higher queue requirements. Furthermore, OBQA significantly improves the performance achieved by the deterministic routing algorithm when no HOL blocking elimination technique is used, especially in hot-spot scenarios. As this is accomplished without requiring many resources, OBQA can be considered as a cost-effective solution to significantly reduce HOL blocking in fat-trees.

\section{Acknowledgements}

This work is jointly supported by the MEC, MICINN and European Commission under projects Consolider Ingenio-2010-CSD2006-00046 and TIN2009-14475-C04, and by the JCCM under projects PCC08-0078 (PhD. grant A08/048) and POII10-0289-3724.

We are grateful to J. Flich, C. Gomez, F. Gilabert, M. E. Gomez y P. Lopez, from the Department of Computer Engineering, Technical University of Valencia, Spain, for their generous support to this work.

\section{References}

1. Myrinet 2000, Series Networking (2000), http: / / www.cspi.com/multicomputer/products/ 2000_series_networking/2000_networking.htm

2. InfiniBand Trade Association. InfiniBand Architecture. Specification Volume 1. Release 1.0, http: //www.infinibandta.com/

3. Top 500 List, http: / / www. top500 . org

4. Leiserson, C.E.: Fat-trees: universal networks for hardware-efficient supercomputing. IEEE Transactions on Computers 34(10), 892-901 (1985)

5. Earth Simulator, http://www.jamstec.go.jp/es/en/index.html

6. Gomez, C., Gilabert, F., Gomez, M., Lopez, P., Duato, J.: Deterministic versus adaptive routing in fat-trees. In: Workshop CAC (IPDPS 2007), p. 235 (March 2007)

7. Gomez, M.E., Lopez, P., Duato, J.: A memory-effective routing strategy for regular interconnection networks. In: Proceedings of the 19th IEEE International Parallel and Distributed Processing Symposium (IPDPS 2005), p. 41.2 (April 2005)

8. Karol, M.J., Hluchyj, M.G., Morgan, S.P.: Input versus output queueing on a space-division packet switch. IEEE Trans. on Commun. COM-35, 1347-1356 (1987)

9. Dally, W., Carvey, P., Dennison, L.: Architecture of the Avici terabit switch/router. In: Proc. of 6th Hot Interconnects, pp. 41-50 (1998)

10. Anderson, T., Owicki, S., Saxe, J., Thacker, C.: High-speed switch scheduling for local-area networks. ACM Transactions on Computer Systems 11(4), 319-352 (1993)

11. Tamir, Y., Frazier, G.: Dynamically-allocated multi-queue buffers for vlsi communication switches. IEEE Transactions on Computers 41(6) (June 1992) 
12. Nachiondo, T., Flich, J., Duato, J.: Destination-based HoL blocking elimination. In: Proc. 12th ICPADS, pp. 213-222 (July 2006)

13. García, P.J., Flich, J., Duato, J., Johnson, I., Quiles, F.J., Naven, F.: Efficient, scalable congestion management for interconnection networks. IEEE Micro 26(5), 52-66 (2006)

14. Mora, G., García, P.J., Flich, J., Duato, J.: RECN-IQ: A cost-effective input-queued switch architecture with congestion management. In: Proc. ICPP (2007)

15. Escudero-Sahuquillo, J., García, P.J., Quiles, F.J., Flich, J., Duato, J.: FBICM: Efficient Congestion Management for High-Performance Networks Using Distributed Deterministic Routing. In: Sadayappan, P., Parashar, M., Badrinath, R., Prasanna, V.K. (eds.) HiPC 2008. LNCS, vol. 5374, pp. 503-517. Springer, Heidelberg (2008)

16. Leiserson, C.E., Maggs, B.M.: Communication-efficient parallel algorithms for distributed random-access machines. Algorithmica 3, 53-77 (1988)

17. k-ary n-trees: High Performance Networks for Massively Parallel Architectures. In: Proceedings of International Parallel Processing Symposium (1997)

18. Duato, J., Yalamanchili, S., Ni, L.: Interconnection networks. An engineering approach. Morgan Kaufmann Publishers, San Francisco (2004)

19. Thoziyoor, S., Muralimanohar, N., Ahn, J.H., Jouppi, N.P.: Cacti 5.1. technical report hpl2008-20. Technical report, Hewlett-Packard Development Company (April 2008) 